\title{
A Prospective Study of the Surgical Outcome of Simple Uvulopalatopharyngoplasty (UPPP), UPPP Combined With Genioglossus Advancement or Tongue Base Advancement for Obstructive Sleep Apnea Hypopnea Syndrome Patients With Multilevel Obstruction
}

\author{
Shicai Chen* ${ }^{\star}$ Song Shi* ${ }^{*}$ Yanghui Xia $\cdot$ Minhui Zhu $\cdot$ Caiyun Zhang $\cdot$ Siwen Xia $\cdot$ Hongliang Zheng \\ Department of Otorhinolaryngology-Head and Neck Surgery, Changhai Hospital, Second Military Medical University of PLA, Shanghai, China
}

Notice of Retraction: Chen et al. A Prospective Study of the Surgical Outcome of Simple Uvulopalatopharyngoplasty (UPPP), UPPP Combined With Genioglossus Advancement or Tongue Base Advancement for Obstructive Sleep Apnea Hypopnea Syndrome Patients With Multilevel Obstruction. Clin Exp Otorhinolaryngol. 2015;8(2):136-41.

The following article from Clinical and Experimental Otorhinolaryngology (CEO), "A prospective study of the surgical outcome of simple uvulopalatopharyngoplasty (UPPP), UPPP combined with genioglossus advancement or tongue base advancement for obstructive sleep apnea hypopnea syndrome patients with multilevel obstruction," [1] published on June 2015 has been retracted from publication.

The authors violated the publication ethics by their dual submission and publication to our journal and the ORL; Journal for Oto-Rhino-Laryngology and Its Related Specialties 2014;76(4):179-188: "Changes in sleep characteristics and airway obstruction in OSAHS patients with multi-level obstruction following simple UPPP, UPPP-GA, or UPPP-TBA: a prospective, single-center, parallel group study" [2]. The inclusion patient and study design, and most results and tables were the same in papers published in both the journals.

Therefore, the editorial board and publication ethics committee of the CEO decided to retract this paper from our journal. We apologize to readers and try the best to thoroughly screen any plagiarisms and ethics violations prior to the publications of papers submitted and accepted in our journal.

Jong-Lyel Roh, MD, PhD

The Editor-in-Chief,

Clinical and Experimental Otorhinolaryngology

\section{REFERENCES}

1. Chen S, Shi S, Xia Y, Zhu M, Zhang C, Xia S, Zheng H. A prospective study of the surgical outcome of simple uvulopalatopharyngoplasty (UPPP), UPPP combined with genioglossus advancement or tongue base advancement for obstructive sleep apnea hypopnea syndrome patients with multilevel obstruction. Clin Exp Otorhinolaryngol. 2015 Jun;8(2):136-41.

2. Chen S, Shi S, Xia Y, Liu F, Chen D, Zhu M, Li M, Zheng H. Changes in sleep characteristics and airway obstruction in OSAHS patients with multi-level obstruction following simple UPPP, UPPP-GA, or UPPP-TBA: a prospective, single-center, parallel group study. ORL J Otorhinolaryngol Relat Spec. 2014;76(4):179-88.

Copyright $\odot 2016$ by Korean Society of Otorhinolaryngology-Head and Neck Surgery.

This is an open-access article distributed under the terms of the Creative Commons Attribution Non-Commercial License (http://creativecommons.org/licenses/by-nc/4.0)

which permits unrestricted non-commercial use, distribution, and reproduction in any medium, provided the original work is properly cited. 